\title{
Plasmons in doped finite carbon nanotubes and their interactions with fast electrons and quantum emitters
}

\author{
Sandra de Vega, ${ }^{1}$ Joel D. Cox ${ }^{1}$ and F. Javier García de Abajo ${ }^{1,2, *}$ \\ ${ }^{1}$ ICFO-Institut de Ciencies Fotoniques, The Barcelona Institute of \\ Science and Technology, 08860 Castelldefels (Barcelona), Spain \\ ${ }^{2}$ ICREA-Institució Catalana de Recerca i Estudis Avançats, \\ Passeig Lluís Companys, 23, 08010 Barcelona, Spain
}

(Dated: August 11, 2016)

\begin{abstract}
We study the potential of highly-doped finite carbon nanotubes to serve as plasmonic elements that mediate the interaction between quantum emitters. Similar to graphene, nanotubes support intense plasmons that can be modulated by varying their level of electrical doping. These excitations exhibit large interaction with light and electron beams, as revealed upon examination of the corresponding light extinction cross-section and electron energy-loss spectra. We show that quantum emitters experience record-high Purcell factors, while they undergo strong mutual interaction mediated by their coupling to the tube plasmons. Our results show the potential of doped finite nanotubes as tunable plasmonic materials for quantum optics applications.
\end{abstract}

PACS numbers: 73.20.Mf, 78.67.Ch, 78.67.Wj, 42.25.Bs

\section{INTRODUCTION}

Plasmons, the collective oscillations of electrons in conducting media, have generated great expectations due to their ability to manipulate optical fields on deep subwavelength scales, thus enabling exciting possibilities for future nanophotonic devices. Traditionally, plasmons have been studied in noble metal nanostructures, where their resonance frequencies and optical coupling strengths are determined by the material intrinsic properties, the morphology of the structure, and the dielectric environment [1-3]. Graphene, the atomically thin carbon layer, has emerged as a promising alternative to noble metals for nanoplasmonic applications due to its ability to support electrically tunable plasmon resonances that interact strongly with light [4-7]. The appealing properties of graphene plasmons are now inspiring research on other two-dimensional van der Waals materials [8, 9], as well as on carbon-based molecules [10, 11]. Currently explored applications of plasmons, including sensing [12-15], photodetection [16-19], wave guiding [20, 21], photovoltaics [22], and medicine [23], could benefit from the identification of new types of actively tunable plasmonic materials with lower inelastic losses.

In a related context, significant advances in quantum information have been gained through the study of coupling between quantum emitters (QEs) and surface plasmons (SPs) supported by noble metals [24-27] or graphitic nanostructures [28, 29]. In particular, carbon nanotubes (CNTs), which are 1D carbon structures formed upon cylindrical wrapping of a graphene sheet, could serve as conduits in plasmonic circuits [30], while their plasmons have been predicted to undergo ultraefficient coupling with QEs [31-35]. Although numer-

*Electronic address: javier.garciadeabajo@icfo.es ous studies on the appealing structural, electrical, and optical properties of CNTs indicate their suitability as components in nanophotonic devices [30, 34, 36, 37], the tunable, low-energy plasmons supported by these structures when they are doped are only now beginning to be explored [7, 37, 38].

In this paper, we investigate the optical response associated with the plasmons of highly-doped finite CNTs, described either by classical electrodynamic or quantummechanical (QM) simulations, and discuss their potential ability to mediate quantum optical interactions on the nanoscale. While plasmons in large-scale graphene nanostructures are currently limited to mid-infrared and lower frequencies, a reduction in their size to the limit represented by polycyclic aromatic hydrocarbons $(\mathrm{PAH})$ can blue-shift resonances toward the visible regime $[10$, 11]. We envision finite CNTs as an intermediate situation between large-scale graphitic structures and $\mathrm{PAH}$ molecules, and therefore as good candidates to extend the robustness and tunability of graphene plasmons toward the visible and near-infrared parts of the spectrum. We also demonstrate that plasmons in finite CNTs can interact strongly with a neighboring QE, while interactions between emitters can be enhanced when they are mediated by $1 \mathrm{D}$ plasmons.

\section{PLASMONS IN DOPED CARBON NANOTUBES}

We first adopt a classical electromagnetic description of doped CNTs by treating them as rolled-up sheets of thickness $t$ and diameter $D$, taking their surface conductivity as that of extended graphene. We describe the level of doping through the change in Fermi energy $E_{\mathrm{F}}$ relative to the neutrality point. Then, as shown in the Supplementary Information (SI) [39], by studying the linear optical response while moving toward the $t \rightarrow 0$ 

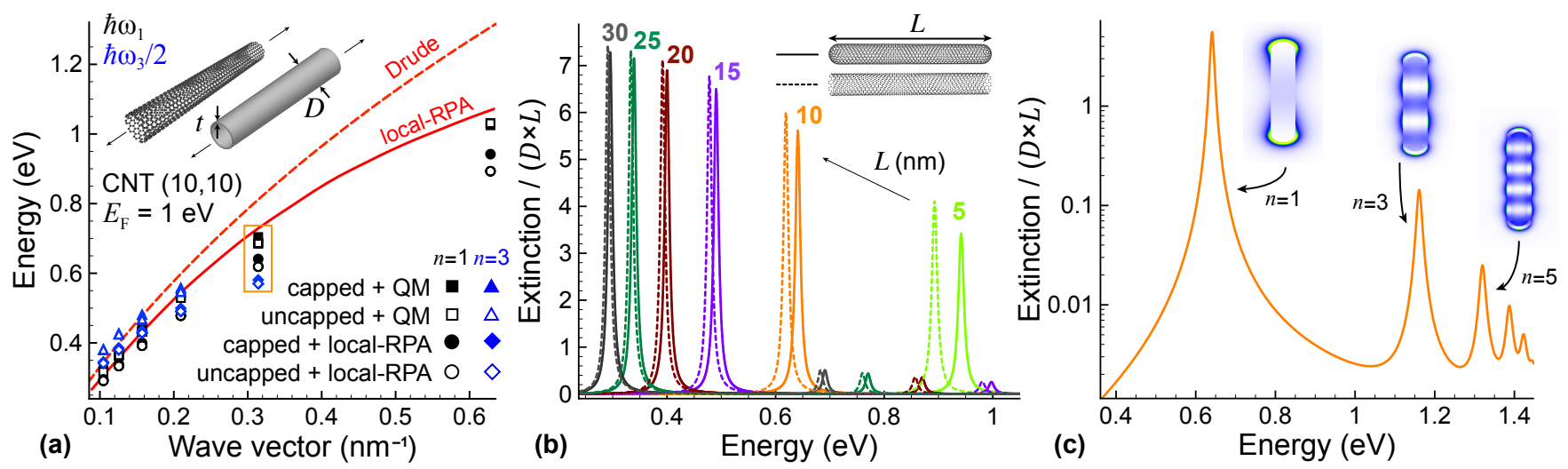

FIG. 1: Plasmon dispersion relation. (a) Comparison of the plasmon dispersion relation for finite and infinite single-wall CNTs. We describe the tubes classically by assuming that the wall responds with the same local 2D surface conductivity $\sigma$ as graphene. Two different models for $\sigma$ are considered (Drude and local-RPA, see labels). The $(10,10)$ CNTs under consideration have a small diameter $\sim 1.35 \mathrm{~nm}$ that only allows the efficient excitation of axially symmetric modes. The plasmon dispersion for infinite tubes is obtained from Eq. (1) (curves). We consider capped and uncapped finite tubes of length $L$, which display modes of different order $n$ dominated by a wave vector $n \pi / L$ (symbols). (b) Extinction cross-section for capped (solid curves) and uncapped (dashes curves) CNTs described with the local-RPA conductivity. (c) Overview of the extinction spectrum for the $L=10 \mathrm{~nm}$ CNT, along with near-field plots for the different plasmon modes. The Fermi energy is $1 \mathrm{eV}$ and the damping is $10 \mathrm{meV}$ in all cases.

limit, we observe converged resonances for $t \sim 0.3 \mathrm{~nm}$, which is similar to the interlayer separation of graphite [see Fig. S1 in the SI [39]]. For finite CNTs of length $L$ larger than the Fermi wavelength $\lambda_{\mathrm{F}}=2 \pi \hbar v_{\mathrm{F}} / E_{\mathrm{F}}$ (e.g., $\lambda_{\mathrm{F}} \sim 4 \mathrm{~nm}$ for $\left.E_{\mathrm{F}}=1 \mathrm{eV}\right)$, the conductivity can be approximated in the local limit. In particular, starting from the random-phase approximation (RPA) [40], we reach the so-called local-RPA surface conductivity, which reduces to the Drude model $\sigma(\omega)=\mathrm{i} e^{2} E_{\mathrm{F}} / \pi \hbar^{2}\left(\omega+\mathrm{i} \tau^{-1}\right)$ for photon energies smaller than $2 E_{\mathrm{F}}$ (i.e., below the threshold for interband transitions).

For simplicity, we concentrate on $(10,10)$ armchair nanotubes (diameter $D=1.35 \mathrm{~nm}$ ) and limit our study to the axially symmetric modes (azimuthal number $m=0$ ), as higher-order plasmons are too tightly confined, and therefore, we expect them to have a weak interaction with incident light and QEs. As a reference, we first simulate CNTs of infinite length, whose plasmon bands are determined by the poles of their reflection coefficient toward cylindrical waves [35], which yield the dispersion relation

$$
\frac{\omega}{\sigma(\omega)}=-4 \pi \mathrm{i} k_{\|}^{2} a I_{0}\left(k_{\|} a\right) K_{0}\left(k_{\|} a\right) .
$$

Here $a=D / 2$ is the tube radius and $I_{0}\left(K_{0}\right)$ is the modified Bessel function of the first (second) kind. We plot in Fig. 1(a) the resulting $m=0$ band, assuming either the Drude model or the local-RPA conductivity. The latter is observed to be redshifted relative to the former, presumably as a result of attractive polarization associated with virtual interband transitions.

In finite CNTs, the plasmon modes can be understood as Fabry-Perot (FP) resonances involving successive reflections at the tube edges. We investigate finite tubes by taking the incident electric field along the symmetry axis and simulating their classical electromagnetic response using the boundary-element method [41]. The resulting extinction spectra are shown in Fig. 1(b), as obtained by using the local-RPA conductivity as obtained by using the local-RPA for either capped (solid curves) or uncapped (broken curves) CNTs of different lengths. As expected from the FP character of the plasmon resonances, the mode frequencies are observed to decrease with increasing length. Additionally, the spectral widths are inherited from the phenomenological lifetime $\tau \approx 66 \mathrm{fs}$ (i.e., $\hbar \tau^{-1}=10 \mathrm{meV}$ ) that we incorporate in the conductivity, as radiative losses are negligibly small because $L$ is much smaller than the light wavelength. As the external field is polarized along the tube axes, only odd-order modes are excited. This is clearly illustrated in Fig. 1(c), which shows an overview of the extinction spectrum of the $L=10 \mathrm{~nm}$ tube for a wide frequency range, where multiple resonances are observed, labeled by the number of nodes in their near-electric-field intensity distributions, $n=1,3, \ldots$ [see insets to Fig. $1(\mathrm{c})]$. The reduction in the maximum cross-section associated with each of the resonances for increasing $n$ is understood from the sign cancellations occurring in their respective induced charges. Noticing that the mode intensity appears to have a maximum at the tube edges, we can approximate the reflection of an $m=0$ plasmon propagating along the tube as 1 (i.e., neglecting losses and assuming zero phase change). Then, the plasmon parallel wave-vector $k_{\|}$must satisfy the condition $k_{\|}=n \pi / L$ in the FP model. The resulting combinations of mode energy and $k_{\|}$observed for different $n$ 's with various tube lengths are represented in Fig. 1(a), in good agreement with the plasmon dispersion 
relation of the infinite tube, thus corroborating the validity of the FP model to qualitatively understand these plasmons.

We now compare these results with those obtained from a QM simulation of the CNTs. Specifically, we use linear response theory within the RPA using electronic states described by a nearest-neighbors tight-binding model with the same parameters as in previous studies of graphene [42-44] (i.e., a single p orbital per carbon site with a hopping energy of $2.8 \mathrm{eV}$ ). As shown in Fig. 1(a), the obtained results are similar to those of the classical description, including the frequency increase associated with larger wave vectors (i.e., smaller $L$ ), as well as the blue shift in the plasmons of capped tubes compared with uncapped tubes. However, the QM model predicts slightly higher-energy plasmons, presumably as a result of quantum confinement, in agreement with previous results for graphene nanodisks [44].

We quantify the strength of the modes by the area of the plasmon features in the extinction spectrum through the $f$-sum rule, $\int_{0}^{\infty} d \omega \sigma^{\text {ext }}(\omega)=2 \pi^{2} e^{2} N_{e} /\left(m_{e}^{*} c\right)$ [45]. Typically, in either classical or QM simulations, we observe that the first mode carries most of the spectral weight (over 80\%), except for the smallest CNT considered (length $L=5 \mathrm{~nm}$ ), in which the first mode takes $\sim 65 \%$ of the total area (see Figs. S1 and S3 in the SI [39]).

As we work in the nonretarded regime, we construct the optical response for CNTs from the electrostatic potential. This allows us to perform an expansion in the eigen-modes of the electric field [7], from which we find a scaling law for the polarizability by designating the diameter $D$ as the characteristic size of a $\mathrm{CNT}$ :

$$
\alpha(\omega)=D^{3} \sum_{j} \frac{A_{j}}{\frac{1}{\eta}-\frac{1}{\eta_{j}}} .
$$

In the above expression, $\eta=\mathrm{i} \sigma(\omega) / \omega D$, while $A_{j}$ and $\eta_{j}$ are size- and material-independent fitting parameters which follow the sum rules $\sum_{j} A_{j}=A / D^{2}$ and $-\sum_{j} \eta_{j} A_{j}=\alpha_{0} / D^{3}$, where $A=L D$ is the crosssectional area of the tube and $\alpha_{0}$ is the polarizability of a perfect-conductor cylinder with the same shape as the CNT (i.e., length $L$ and diameter $D$ ). Through the optical theorem $\sigma^{\text {ext }}(\omega)=4 \pi(\omega / c) \operatorname{Im}\{\alpha(\omega)\}$, we find an expression to fit the resonances shown in Fig. 1(b), from which we obtain values for $A_{j}$ and $\eta_{j}$ (see Fig. S2 in the SI [39]) as a function of both thickness and length.

\section{INTERACTION WITH FOCUSED ELECTRON BEAMS}

We now shift our attention to the interaction of CNT plasmons with energetic electrons as a means to investigate their spatial and spectral characteristics. Indeed, many of the properties of plasmons have been revealed by electron-microscope spectroscopies [46], which are better suited than optics-based methods to probe these deep-subwavelength excitations. In particular, electron energy-loss spectroscopy (EELS) appears to be an ideal technique to study the strongly confined plasmons of CNTs. We thus present in Fig. 2 results for $10 \mathrm{keV}$ electrons passing near a CNT along the two trajectories shown in the insets. We find that different plasmon modes are excited with different strength, also depending on the orientation of the trajectory. For example, highorder modes (i.e., above the energy range shown in Fig. 2) are more easily excited in the trajectory that runs parallel to the tube, while the perpendicular trajectory is more efficient at exciting the lowest-order $\sim 0.65 \mathrm{eV}$ mode. In particular, the dipolar plasmon has its field concentrated near the tube ends, thus favoring the interaction with the perpendicular trajectory under consideration.

Our choice of a $1 \mathrm{~nm}$ beam-CNT separation is not critical, as we find the EELS probability for excitation of the dipolar plasmon to decay exponentially with a characteristic distance $\sim L / 2 \pi$ (see Fig. S5 in the SI [39]). This result can be understood as follows: from the above FP model, the plasmon wave vector must be $\sim \pi / L$ along the tube; as the plasmon evolves in the quasistatic limit, its wave vector along the perpendicular direction must be $\sim \mathrm{i} \pi / L$; consequently, we find a dependence $\propto \exp (-2 \pi x / L)$ of the electric-field intensity associated with the plasmon on the distance to the tube $x$, which is directly inherited by the EELS probability. In general, plasmons of order $n$ should decay with a characteristic transversal distance $\sim L / 2 \pi n$.

Remarkably, the peak probability reaches high values, comparable to or larger than those typically encountered in EELS studies of larger metal nanoparticles. We find that the number of plasmons excited per electron (i.e., the integrated peak area, which should be roughly independent of $\tau$ ) can reach $0.1 \%$, although this number scales as $\sim 1 / v^{2}$ with electron velocity $v$ for small objects (cf. $v / c=0.19$ for the $10 \mathrm{keV}$ electrons here considered and $v / c=0.55$ for $100 \mathrm{keV})$.

\section{INTERACTION WITH QUANTUM EMITTERS}

Light can be confined to extremely small regions of space through the excitation of localized surface plasmons, thus presenting an opportunity to achieve strong coupling between a QE and optical fields. Here we assess the strength of a $\mathrm{QE}$ coupling to the local electromagnetic fields of CNT plasmons through quantitative analysis of the enhancement in its radiative decay rate. In what follows, we represent a QE as a two-level system characterized by its transition dipole moment $\mathbf{d}$. If a single QE is placed in close proximity to a CNT, it induces a field $\mathbf{E}^{\text {ind }}$ from the CNT that acts back on the emitter, thereby modifying its decay rate according to [47]

$$
\Gamma_{11}(\omega)=\Gamma_{0}(\omega)+\frac{2}{\hbar} \operatorname{Im}\left\{\mathbf{d}^{*} \cdot \mathbf{E}^{\text {ind }}\right\},
$$




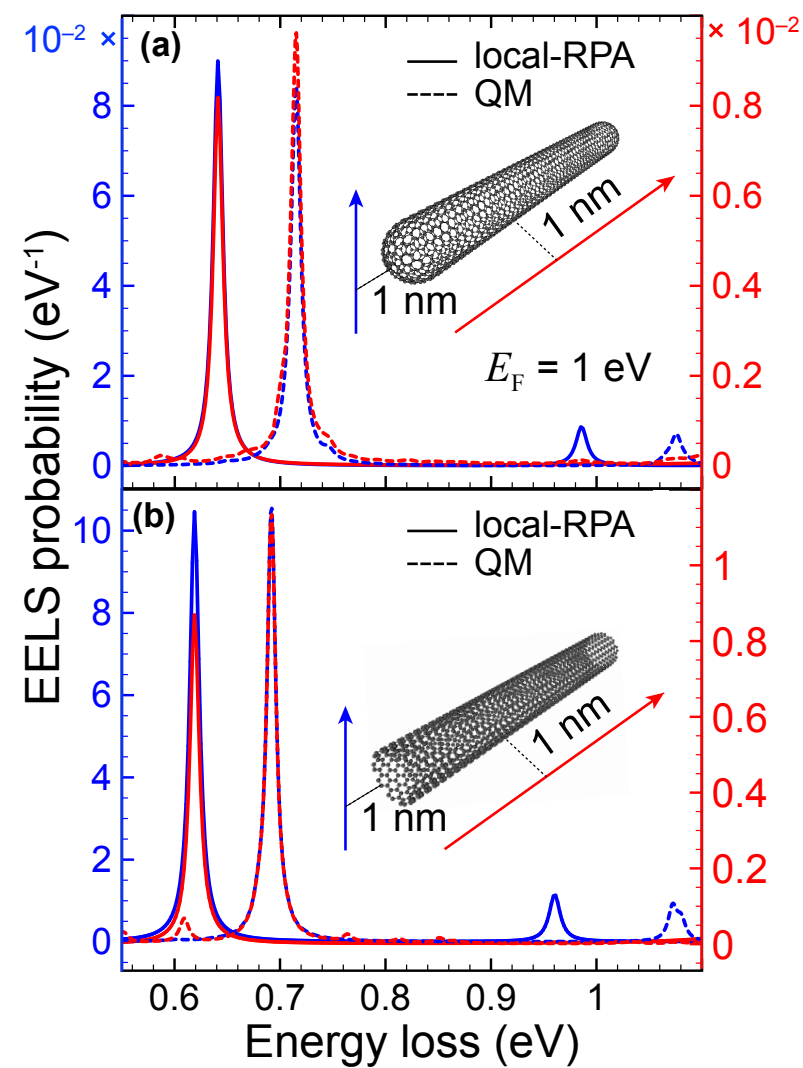

FIG. 2: Plasmon excitation with electron beams. (a) Electron energy-loss probability for a $10 \mathrm{keV}$ electron passing near a $L=10 \mathrm{~nm}$ capped $(10,10)$ CNT along two different trajectories (see inset). Classical local-RPA calculations (solid curves) are compared with quantum RPA simulations (broken curves). (b) Same as (a) for an uncapped tube.

where $\Gamma_{0}(\omega)=4 \omega^{3}|\mathbf{d}|^{2} / 3 \hbar c^{3}$ is the decay rate in free space. If we now consider two such QEs that are placed at opposite ends of the CNT (see inset to Fig. 3), we can quantify their interaction mediated by the CNT by studying the radiative decay rate enhancement of the first $\mathrm{QE}$ (e.g., the QE at position 1) due to the induced electric field $\mathbf{E}_{12}$ produced on it by the second QE (e.g., the QE at position 2) as [48]

$$
\Gamma_{12}(\omega)=\frac{2}{\hbar} \operatorname{Im}\left\{\mathbf{d}_{1}^{*} \cdot \mathbf{E}_{12}\right\} .
$$

We remark that under the conditions considered here our results indicate that the natural decay rate $\Gamma_{0}$ is much smaller than the enhanced decay rate (see below). Then, assuming for simplicity that the QEs are identical, the symmetry of the CNT and its plasmon modes allows us to approximate $\Gamma_{11} \simeq \Gamma_{12}$ when the QEs are placed on opposite ends of the carbon structure.

In Fig. 3(a) we show $\Gamma_{i j} / \Gamma_{0}(i, j=1,2)$ for two QEs, each located at a distance of one nanometer from either ends of a $10 \mathrm{~nm}$-long $(10,10)$ CNT (red curves correspond to uncapped CNTs and black curves to capped CNTs).
Remarkably, we observe an enhancement of the order of $\sim 10^{8}$, which is several orders of magnitude higher than predicted for graphene disks [49]. This strong interaction is a consequence of the fact that the emitter decays with close to unity probability into the CNT plasmon (instead of into other inelastic decay channels such as electronhole pairs), even when placed at such a short distance from the tube, as previously shown for extended CNTs [35]. Additionally, these results do not depend critically on the exact positioning of the QEs: we expect their interaction to roughly scale with the product of the plasmon field amplitudes at the position of the emitters; this amplitude decays exponentially over a distance of several nanometers (see the above discussion on the distance dependence of the EELS probability). This intuition is corroborated by Fig. S4 of the SI [39], where we present numerical results for different QE-CNT distances (including asymmetrical configurations).

Now, invoking the Jaynes-Cummings model [50], such high Purcell factors should enable quantum entanglement between the emitters [51, 52], as well as the implementation of logical gates [26], thus providing further motivation for the application of doped CNTs in nanoscale quantum devices.

Additionally, the real part of the interaction between the $\mathrm{QE}$ and the induced field of the CNT yields the energy splitting of the QE states [Fig. 3(b)], which is quantified by

$$
G_{i j}(\omega)=\frac{1}{\hbar} \operatorname{Re}\left\{\mathbf{d}_{i}^{*} \cdot \mathbf{E}_{i j}\right\},
$$

where $i, j=1,2$ indicate the QE positions. This interaction is a few times lower in magnitude than $\Gamma_{i j}$, therefore indicating that one could reach a regime in which the decay rate of fast emitters $(\mathrm{GHz})$ is made close to their optical frequency $(\mathrm{PHz})$, with a small fractional correction in emission frequency.

Figure 3(c) shows the dependence of the Purcell factor on the electron mobility, which enters both the classical and QM models through the phenomenological relaxation rate $\tau$. Even for extremely low plasmon lifetimes $\tau$ of the order of $\sim 10 \mathrm{fs}$, doped CNTs are predicted to enable Purcell factors $\sim 10^{7}$, while we observe a linear dependence of such factors on $\tau$. These results are very similar within both classical and quantum-mechanical models, thus corroborating once more the validity of the former to cope with structures that contain only several thousand atoms.

\section{CONCLUSION}

We have demonstrated that highly-doped finite CNTs exhibit intense plasmon resonances that can mediate the interaction between quantum emitters placed in their proximity. In particular, CNT plasmons are found to 

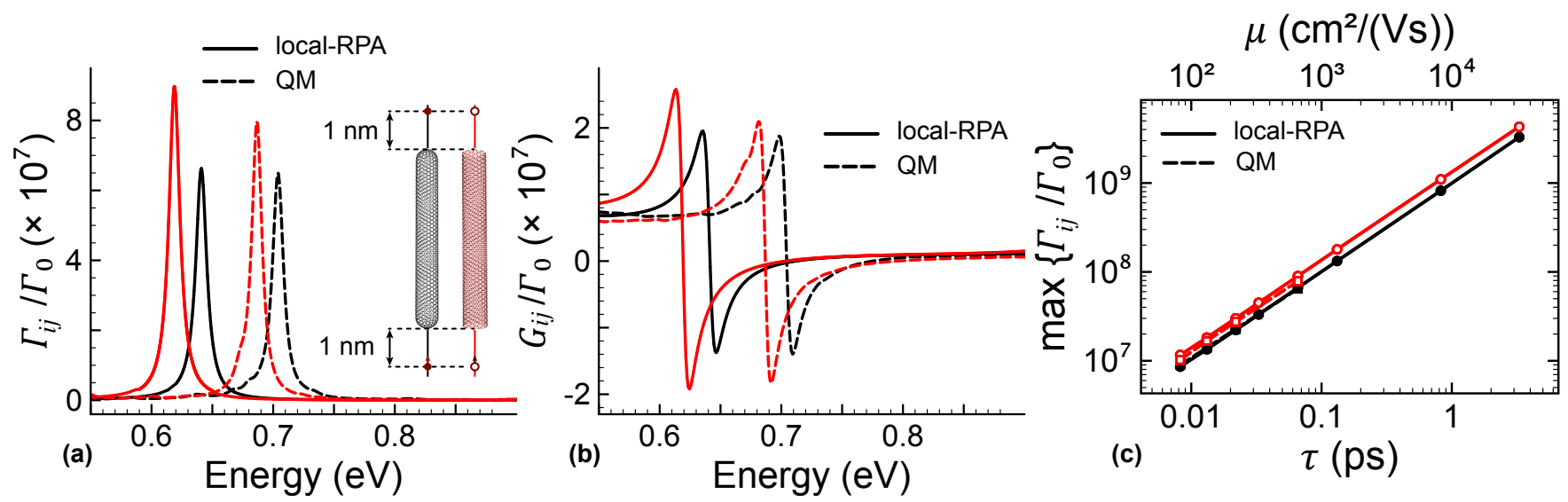

FIG. 3: Interaction with quantum emitters. (a,b) Emission-energy dependence of the imaginary (a) and real (b) parts of the QE interactions, normalized to the free-space emission rate $\Gamma_{0}$. We consider identical, axially polarized QEs placed $1 \mathrm{~nm}$ away from the surface of a 10-nm-long $(10,10)$ CNT with either capped (black) and uncapped (red) ends. The Purcell factor $\Gamma_{j j} / \Gamma_{0}$ cannot be separated from the interemitter interaction $\Gamma_{12} / \Gamma_{0}$ on the scale of the plot. We compare classical local-RPA (solid curves) and quantum RPA (broken curves) calculations for a Fermi energy of $1 \mathrm{eV}$ and a damping of $10 \mathrm{meV}$. (b) Dependence of the interemitter interaction on the plasmon relaxation time $\tau$. Solid black (open red) symbols account for maxima of the Purcell factor given by a capped (uncapped) CNT. Classical local-RPA (quantum-mechanical) calculations correspond to the circles (squares). The equivalent mobility $\mu=\tau e v_{\mathrm{F}}^{2} / E_{\mathrm{F}}$ within the Drude model is shown in the upper horizontal scale.

produce optical extinction cross-sections exceeding the CNT projected areas, with Purcell factors for proximal quantum emitters reaching $\sim 10^{8}$, enabling strong interaction between emitters located at opposite ends of a CNT. These plasmons lie in the near-infrared part of the spectrum for realistic tube dimensions, thus holding potential for their use in technological applications within that frequency range. We introduce a classical electromagnetic description of the CNT optical response that predicts plasmon energies and coupling strengths in good agreement with those obtained from an atomistic model (tight-binding description of the electronic states combined with RPA linear response) both in the case of capped and uncapped CNTs. Our results indicate a strong potential for highly-doped CNTs as robust, actively tunable plasmonic elements that are well-suited for nanophotonic and quantum-optics applications.

\section{Acknowledgments}

This work has been supported in part by the Spanish MINECO (MAT2014-59096-P and SEV2015-0522) and the European Commission (Graphene Flagship CNECTICT-604391 and FP7-ICT-2013-613024-GRASP). SVE acknowledges financial support through FPU from the Spanish MECD.
[1] J. Crowell and R. H. Ritchie, Phys. Rev. 172, 436 (1968).

[2] W. L. Barnes, A. Dereux, and T. W. Ebbesen, Nature 424, 824 (2003).

[3] B. Diaconescu, K. Pohl, L. Vattuone, L. Savio, P. Hofmann, V. M. Silkin, J. M. Pitarke, E. V. Chulkov., P. M. Echenique, D. Farías, et al., Nature 448, 57 (2007).

[4] A. K. Geim and K. S. Novoselov, Nat. Mater. 6, 183 (2007).

[5] M. Jablan, H. Buljan, and M. Soljačić, Phys. Rev. B 80, 245435 (2009).

[6] A. N. Grigorenko, M. Polini, and K. S. Novoselov, Nat. Photon. 6, 749 (2012).

[7] F. J. García de Abajo, ACS Photon. 1, 135 (2014).

[8] C. R. Dean, A. F. Young, I. Meric, C. Lee, L. Wang, S. Sorgenfrei, K. Watanabe, T. Taniguchi, P. Kim, K. L. Shepard, et al., Nat. Nanotech. 5, 722 (2010).
[9] A. Principi, M. Carrega, M. B. Lundeberg, A. Woessner, F. H. Koppens, G. Vignale, and M. Polini, Phys. Rev. B 90, 165408 (2014).

[10] A. Manjavacas, F. Marchesin, S. Thongrattanasiri, P. Koval, P. Nordlander, D. Sánchez-Portal, and F. J. García de Abajo, ACS Nano 7, 3635 (2013).

[11] A. Lauchner, A. Schlather, A. Manjavacas, Y. Cui, M. J. McClain, G. J. Stec, F. J. García de Abajo, P. Nordlander, , and N. J. Halas, Nano Lett. 15, 6208 (2015).

[12] S. Enoch, R. Quidant, and G. Badenes, Opt. Express 29, 3422 (2004).

[13] S. A. Maier, M. D. Friedman, P. E. Barclay, and O. Painter, Appl. Phys. Lett. 86, 071103 (2005).

[14] J. N. Anker, W. P. Hall, O. Lyandres, N. C. Shah, J. Zhao, and R. P. Van Duyne, Nat. Mater. 7, 442 (2008).

[15] A. Marini, I. Silveiro, and F. J. García de Abajo, ACS 
Photon. 2, 876 (2015).

[16] F. D. Angelis, M. Patrini, G. Das, I. Maksymov, M. Galli, L. Businaro, L. Andreani, and E. D. Fabrizio, Nano Lett. 8, 2321 (2008).

[17] F. Neubrech, A. Pucci, T. W. Cornelius, S. Karim, A. García-Etxarri, and J. Aizpurua, Phys. Rev. Lett. 101, 157403 (2008).

[18] Y. Liu, R. Cheng, L. Liao, H. Zhou, J. Bai, G. Liu, L. Liu, Y. Huang, and X. Duan, Nat. Commun. 2, 579 (2011).

[19] H. Chalabi, D. Schoen, and M. L. Brongersma, Nano Lett. 14, 1374 (2014).

[20] G. W. Hanson, J. Appl. Phys. 103, 064302 (2009).

[21] A. Manjavacas and F. J. García de Abajo, Nano Lett. 9, 1285 (2009).

[22] H. A. Atwater and A. Polman, Nat. Mater. 9, 205 (2010).

[23] X. Qian, X.-H. Peng, D. O. Ansari, Q. Yin-Goen, G. Z. Chen, D. M. Shin, L. Yang, A. N. Young, M. D. Wang, and S. Nie, Nat. Biotech. 26, 83 (2008).

[24] D. E. Chang, A. S. Sörensen, P. R. Hemmer, and M. D. Lukin, Phys. Rev. Lett. 97, 053002 (2006).

[25] D. E. Chang, A. S. Sørensen, P. R. Hemmer, and M. D. Lukin, Phys. Rev. B 76, 035420 (2007).

[26] D. Dzsotjan, A. S. Sörensen, and M. Fleischhauer, Phys. Rev. B 82, 075427 (2010).

[27] A. Gonzalez-Tudela, D. Martin-Cano, E. Moreno, L. Martín-Moreno, C. Tejedor, and F. J. Garcia-Vidal, Phys. Rev. Lett. 106, 020501 (2011).

[28] P. A. Huidobro, A. Y. Nikitin, C. González-Ballestero, L. Martín-Moreno, and F. J. García-Vidal, Phys. Rev. B 85, 155438 (2012)

[29] F. H. L. Koppens, D. E. Chang, and F. J. García de Abajo, Nano Lett. 11, 3370 (2011).

[30] I. Soto Lamata, P. Alonso-González, R. Hillenbrand, and A. Y. Nikitin, ACS Photon. 90, 125417 (2015).

[31] I. V. Bondarev, G. Y. Slepyan, and S. A. Maksimenko, Phys. Rev. Lett. 89, 115504 (2002).

[32] I. V. Bondarev and B. Vlahovic, Phys. Rev. B 75, 073401 (2006).

[33] I. V. Bondarev and B. Vlahovic, Phys. Rev. B 75, 033402
(2007).

[34] I. V. Bondarev, J. Comput. Theor. Nanosci. 7, 1673 (2010).

[35] L. Martín-Moreno, F. J. García de Abajo, and F. J. García-Vidal, Phys. Rev. Lett. 115, 173601 (2015).

[36] P. Avouris, M. Freitag, and V. Perebeinos, Nat. Phot. 2, 341 (2008).

[37] Z. Shi, X. Hong, H. A. Bechtel, B. Zeng, M. C. Martin, K. Watanabe, T. Taniguchi, Y.-R. Shen, and F. Wang, Nat. Photon. 9, 515 (2015).

[38] T. Morimoto, S.-K. Joung, T. Saito, D. N. Futaba, K. Hata, and T. Okazaki, ACS Nano 8, 9897 (2014).

[39] See supplementary material at http://link.aps.org/supplemental/xxx.

[40] D. Pines and P. Nozières, The Theory of Quantum Liquids (W. A. Benjamin, Inc., New York, 1966).

[41] F. J. García de Abajo and A. Howie, Phys. Rev. B 65, 115418 (2002).

[42] P. R. Wallace, Phys. Rev. 71, 622 (1947).

[43] A. H. Castro Neto, F. Guinea, N. M. R. Peres, K. S. Novoselov, and A. K. Geim, Rev. Mod. Phys. 81, 109 (2009).

[44] S. Thongrattanasiri, A. Manjavacas, and F. J. García de Abajo, ACS Nano 6, 1766 (2012).

[45] J. J. Sakurai, Modern Quantum Mechanics (AddisonWesley, 1994).

[46] F. J. García de Abajo, Rev. Mod. Phys. 82, 209 (2010).

[47] L. Novotny and B. Hecht, Principles of Nano-Optics (Cambridge University Press, New York, 2006).

[48] Z. Ficek and R. Tanas, Phys. Rep. 372, 369 (2002).

[49] A. Manjavacas, S. Thongrattanasiri, D. E. Chang, and F. J. García de Abajo, New J. Phys. 14, 123020 (2012).

[50] E. Jaynes and F. Cummings, Proc. IEEE 51, 89 (1963).

[51] P. Meystre and M. Sargent, Elements of Quantum Optics (Springer-Verlag, Berlin, 2007).

[52] L. M. Duan and C. Monroe (Academic Press, 2008), vol. 55 of Advances In Atomic, Molecular, and Optical Physics, pp. 419-463. 


\title{
Supplementary Information for
}

"Plasmons in doped finite carbon nanotubes and their interactions with fast electrons and quantum emitters"

\author{
Sandra de Vega, ${ }^{1}$ Joel D. Cox ${ }^{1}$ and F. Javier García de Abajo ${ }^{1,2, *}$ \\ ${ }^{1}$ ICFO - Institut de Ciències Fotòniques, Mediterranean Technology Park, 08860 Castelldefels (Barcelona), Spain \\ ${ }^{2}$ ICREA - Institució Catalana de Recerca i Estudis Avançats, \\ Passeig Lluís Companys, 23, 08010 Barcelona, Spain
}

(Dated: August 11, 2016)

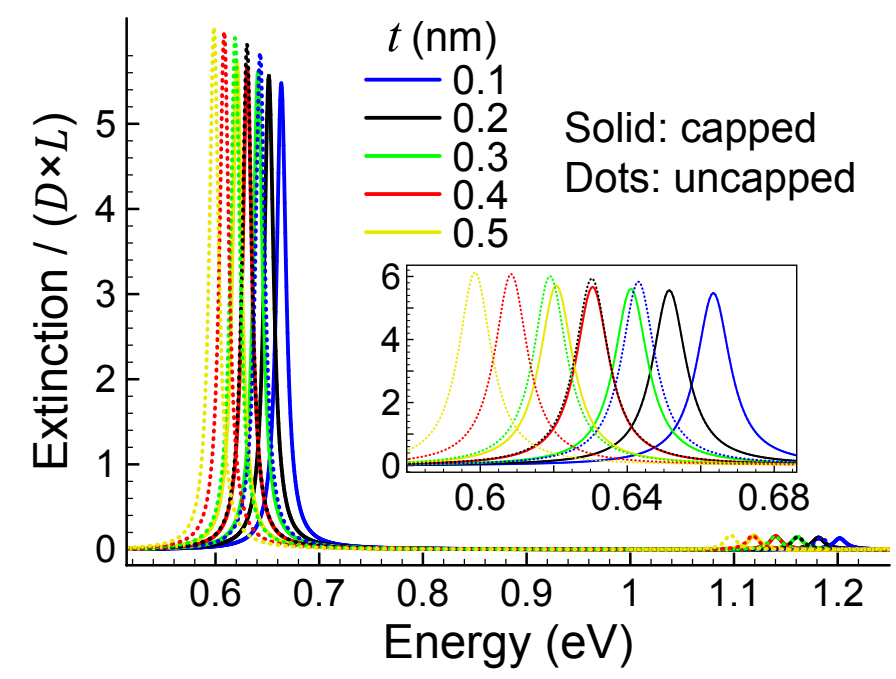

FIG. S1: Dependence of the optical extinction on carbon-wall thickness. Extinction cross-section of a CNT(10,10) normalized to the projected area $D L$, as calculated within a classical model using the local RPA graphene conductivity. Results are presented for different thicknesses and a fixed length of $10 \mathrm{~nm}$. The inset shows a zoom of the dominant plasmon resonance. Solid (broken) curves correspond to capped (uncapped) CNTs.
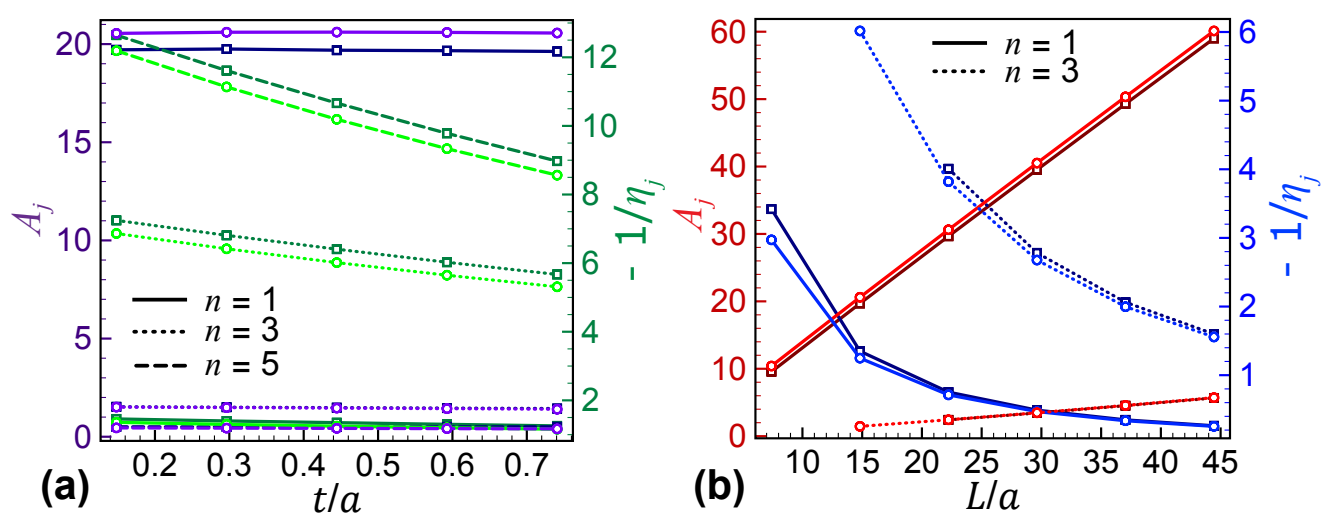

FIG. S2: Scaling law parameters. (a) CNT-thickness dependence of the scaling law parameters [see Eq. (2) in the main text]. Dark (light) colors with squares (circles) correspond to capped (uncapped) CNTs. (b) CNT-length dependence.

*Electronic address: javier.garciadeabajo@icfo.es 


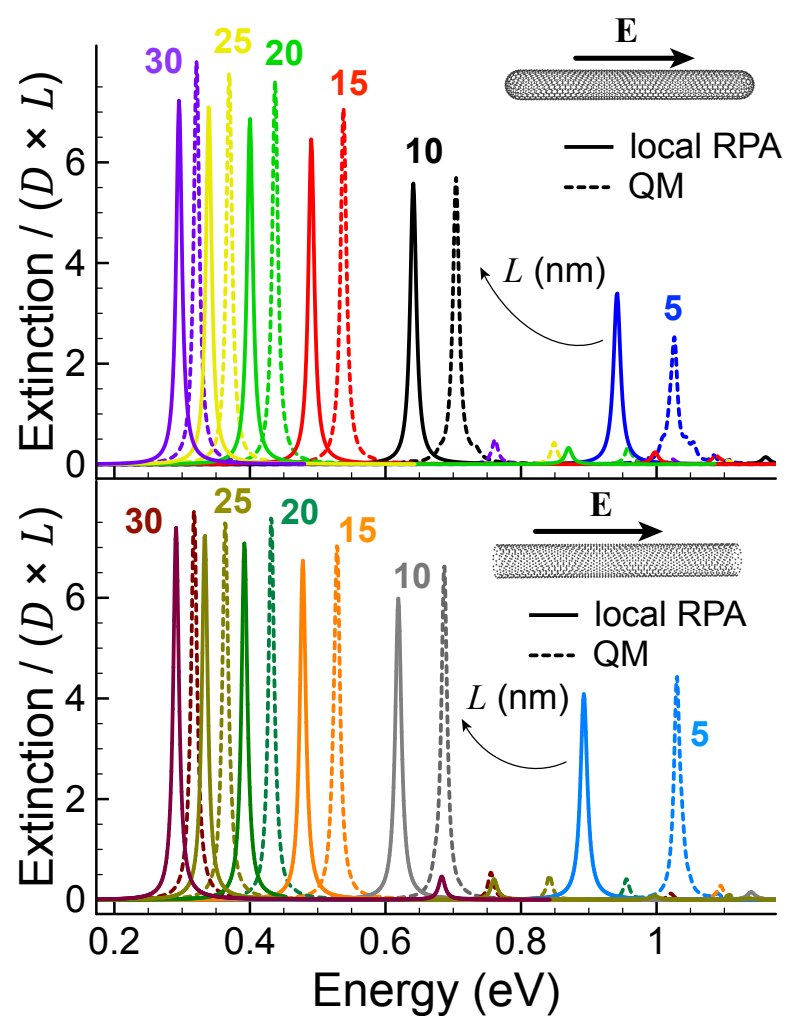

FIG. S3: Optical extinction for different response models. Extinction cross-section for different CNT(10,10) lengths $L$ obtained from two different response models: classical description with the local RPA graphene conductivity (solid curves) and quantum-mechanical atomistic model based on the RPA (dashed curves). 


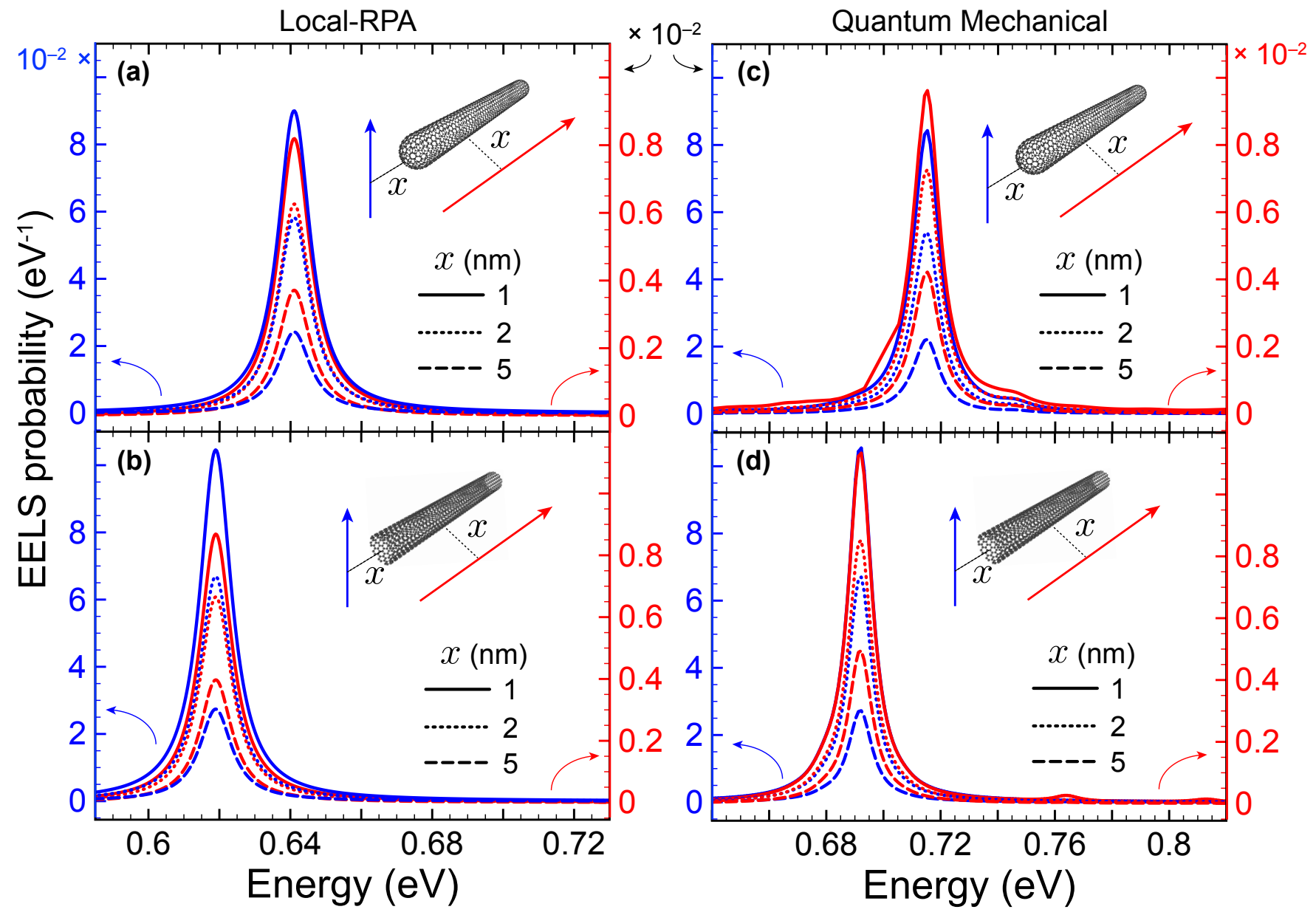

FIG. S4: Distance dependence of the interaction with QEs. We compare the coupling coefficients shown in Fig. 3(a) of the main paper, and reproduced in panel (a) of this figure for convenience, with those obtained by moving the QEs away from the tube ends, either in asymmetric (b) or symmetric (c) configurations.

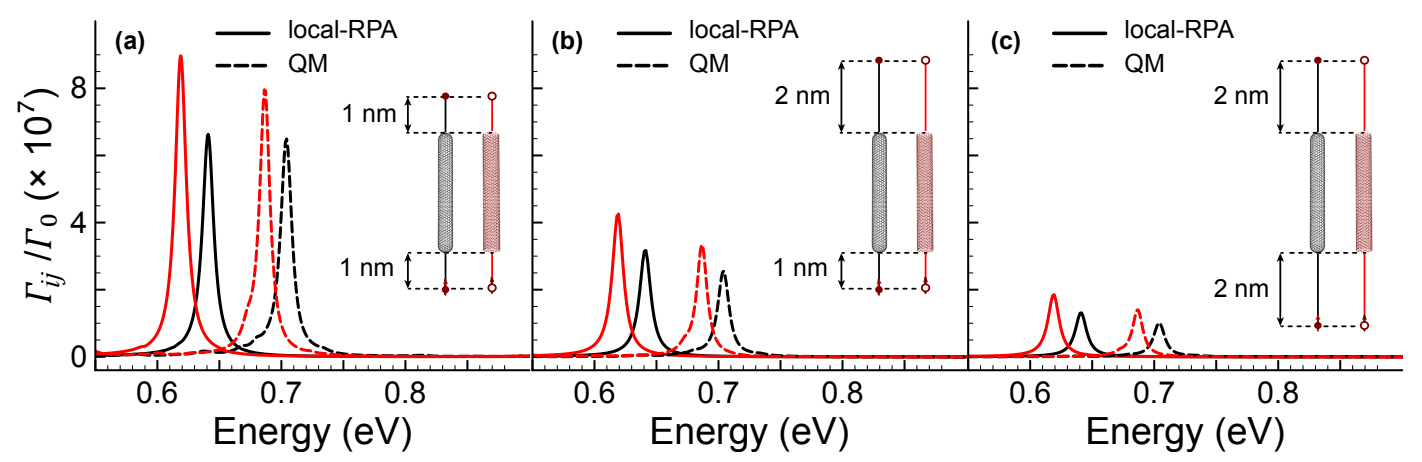

FIG. S5: Dependence of the EELS probability on electron-beam-CNT distance. We show classical local-RPA (left panels) and quantum-mechanical (right panels, tight binding plus RPA) calculations of the EELS probability under the same conditions as in Fig. 2 of the main paper with different electron-beam-CNT separations for capped (upper panels) and uncapped (lower panels) CNTs. 